\title{
The dynamical effect of white dwarf kicks in star clusters
}

\author{
Harvey B. Richer \\ Department of Physics and Astronomy, University of British Columbia, \\ 6224 Agricultural Road, Vancouver, British Columbia, Canada V6T 1Z1 \\ email: richer@astro.ubc.ca
}

\begin{abstract}
Globular star clusters generally have large cores, i.e., $r_{\mathrm{c}} / r_{\mathrm{h}}$ (the ratio of core to halflight radii) exceeds 0.3 for more than $50 \%$ of the Galactic globular clusters. In the absence of a central heating source, dynamical models suggest that massive clusters will contract, typically on a timescale shorter than a Hubble time, and exhibit a compact core. To explain the disagreement between observations and theory, intermediate-mass mass black holes have been invoked to explain the core structure. Recent observations, however, have failed to definitively prove their existence in clusters. A new scenario, involving a natal kick given to white dwarfs may provide the required heating and help clusters avoid or delay core collapse.
\end{abstract}

Keywords. globular clusters: general, white dwarfs, black hole physics, stellar dynamics

\section{Introduction}

An examination of the distribution of core to half-light radii $\left(r_{\mathrm{c}} / r_{\mathrm{h}}\right)$ in globular star clusters indicates that many clusters have large core radii relative to their half-light radii, fully $50 \%$ have this ratio $>0.3$. So, most clusters do not currently appear to be in a corecollapsed (or binary-burning) phase of evolution. They may still be in the standard initial contraction phase or they may have had their core sizes enhanced through some excess energy source in the cluster. $N$-body or Monte Carlo models (e.g., Heggie et al. 2006) demonstrate that, with zero binaries, a cluster rapidly goes into core collapse and only with an appreciable primordial binary fraction $(>10 \%)$ could core collapse be avoided or delayed for a time comparable to the age of the Universe. Thus, putting an appreciable binary fraction into the models helps bring the theoretical structural picture of globular clusters closer to the real data, although even with $100 \%$ binaries, very large core radii could not be achieved in the dynamical simulations. Thus the structure of the bulk of the globular star clusters cannot be explained simply by assuming a large binary fraction: the cluster must either be dynamically young or have some new energy source.

\section{Intermediate-mass black holes in star clusters}

With binaries clearly not the solution to large cluster cores, the role of intermediatemass black holes (IMBHs) was examined. These are black holes in the mass range from roughly 100 to a few times $10000 \mathrm{M}_{\odot}$. N-body calculations by numerous authors (e.g., Gill et al. 2008; Trenti et al. 2007) showed that large cores could easily be obtained with the presence of an IMBH with a mass a few percent of that of the total cluster. Some authors even made statements to the effect that an IMBH was required in the cores of those clusters exhibiting very large core radii. An observational signature of the presence of an IMBH in a star cluster is an increase in the cluster velocity dispersion near the core (Baumgardt et al. 2005). Several heroic attempts have been made to search 
for this effect in a few clusters and while none of these cases was rock solid, they were certainly suggestive of the presence of IMBHs in some clusters. However, recently two proper-motion studies at the centers of loose clusters (Ruberg 2008; Anderson \& van der Marel 2009) failed to yield any evidence for the presence of IMBHs.

\section{A new heat source: kicked white dwarfs}

A new paradigm seems required to explain the core dynamics in globular star clusters. Davis et al. (2008) showed that in the globular cluster NGC 6397 the young white dwarfs appear to have been given a small 'kick' when they were born, on the order of $3-5 \mathrm{~km}$ $\mathrm{s}^{-1}$. Including such a kick in dynamical models demonstrated that large core radii could easily be obtained in globular clusters and that core collapse could be delayed for a period longer than a Hubble time (Fregeau et al. 2009). How does this explain the large cluster cores? A kicked white dwarf will lose energy to its surrounding stars as it is moving too fast for its mass in equipartition. In this sense, it supplies heat to the cluster, puffing up its core (Heyl 2008).

The physical mechanism for a white-dwarf kick is not immediately apparent. Asymmetric mass loss in the late asymptotic giant branch or planetary-nebula phase seems most obvious, but a kick at the core helium flash may also supply the energy input needed. We are currently investigating this observationally by exploring the radial distributions of stars in clusters in all evolutionary phases beyond the main-sequence turnoff. In addition, we are examining the location of the white dwarf in planetary nebulae to determine whether it is located off center. This could be evidence for a kick late in the evolution of a star.

\section{References}

Anderson, J. \& van der Marel, R. P. 2009, ApJ, submitted (arXiv:0905.0627)

Baumgardt, H., Makino, J., \& Hut, P. 2005, ApJ, 620, 238

Davis, D. S., Richer, H. B., King, I. R., Anderson, J., Coffey, J., Fahlman, G. G., Hurley, J., \& Kalirai, J. S. 2008, MNRAS (Letters), 383, L20

Fregeau, J. M., Richer, H. B., Rasio, F. A., \& Hurley, J. R. 2009, ApJ (Letters), 695, L20

Gill, M., Trenti, M., Miller, M. C., van der Marel, R., Hamilton, D., \& Stiavelli, M. 2008, ApJ, 686,303

Heggie, D. C., Trenti, M., \& Hut, P. 2006, MNRAS, 368, 677

Heyl, J. 2008, MNRAS, 390, 622

Ruberg, A. 2008, MSc Thesis, University of British Columbia

Trenti, M., Ardi, E., Mineshige, S., \& Hut, P. 2007, MNRAS, 374, 857 